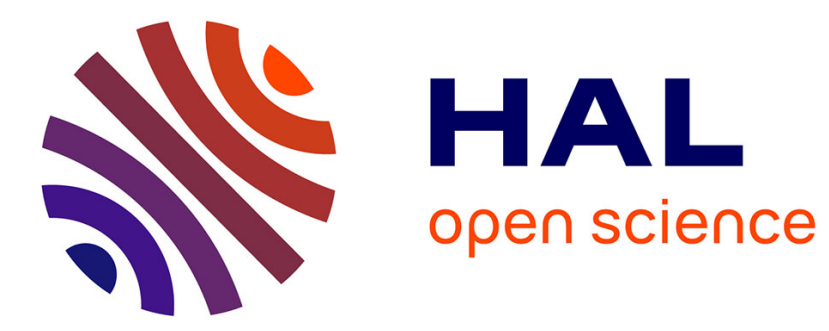

\title{
De la vaisselle et du vin chypriote au Létôon de Xanthos à l'époque romaine
}

Sandrine Elaigne, Séverine Lemaître

\section{To cite this version:}

Sandrine Elaigne, Séverine Lemaître. De la vaisselle et du vin chypriote au Létôon de Xanthos à l'époque romaine. Topoi Orient - Occident, 2014, 19 (2), pp.565 - 593. 10.3406/topoi.2014.2554. halshs-01928090

\section{HAL Id: halshs-01928090 \\ https://shs.hal.science/halshs-01928090}

Submitted on 28 May 2020

HAL is a multi-disciplinary open access archive for the deposit and dissemination of scientific research documents, whether they are published or not. The documents may come from teaching and research institutions in France or abroad, or from public or private research centers.
L'archive ouverte pluridisciplinaire HAL, est destinée au dépôt et à la diffusion de documents scientifiques de niveau recherche, publiés ou non, émanant des établissements d'enseignement et de recherche français ou étrangers, des laboratoires publics ou privés. 
De la vaisselle et du vin chypriote au Létôon de Xanthos à l'époque romaine

Sandrine Elaigne, Séverine Lemaître

\section{Citer ce document / Cite this document :}

Elaigne Sandrine, Lemaître Séverine. De la vaisselle et du vin chypriote au Létôon de Xanthos à l'époque romaine. In: Topoi, volume 19/2, 2014. pp. 565-593;

doi : https://doi.org/10.3406/topoi.2014.2554

https://www.persee.fr/doc/topoi_1161-9473_2014_num_19_2_2554

Fichier pdf généré le 10/12/2019 


\section{DE LA VAISSELLE ET DU VIN CHYPRIOTE AU LÉTÔON DE XANTHOS À L'ÉPOQUE ROMAINE}

Le rôle de Chypre comme carrefour des relations et des échanges au sein du bassin oriental de la Méditerranée depuis au moins le $\mathrm{II}^{\mathrm{e}}$ millénaire avant J.-C. est un sujet largement abordé sous différents aspects: histoire événementielle, culturelle, échanges d'artefacts. Loin de s'être amenuisé, ce rôle semble encore important à l'époque romaine.

Les recherches menées dans le cadre de la mission française de XanthosLétôon ${ }^{1}$ depuis 1995 , en particulier sur les mobiliers céramiques, permettent aujourd'hui d'alimenter, sous un nouvel angle de vue, celui de la côte lycienne, le copieux dossier des liens entre Chypre et les régions voisines (Fig.1). En effet, les sources écrites apportent plusieurs témoignages quant à l'importance du trafic maritime entre la Lycie et Chypre, qui constituait une escale incontournable pour les liaisons entre la côte sud anatolienne et le littoral égyptien, notamment Alexandrie $^{2}$. De plus, peu de doute plane sur la persistance, à l'époque romaine, de la connexion triangulaire existant à l'époque hellénistique, qui reliait Rhodes

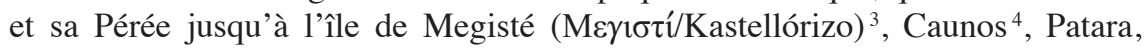

1. La direction de la mission française de Xanthos était assurée par J. des Courtils qui a non seulement permis, mais encouragé le développement des études céramologiques sur les sites de Xanthos et du Létôon. La direction du site du Létôon était alors aux mains de D.Laroche, que nous tenons à remercier pour sa bienveillance à l'égard de notre travail.

2. Arnaud 2005, p. 215-216.

3. Holleaux 1893, p.52; Arnaud 2011.

4. Holleaux 1893, pp.61-69. Rougé 1966, p.209, 448-449. Robert 1987, p.509, sur l'exemption de droits concernant importations et exportations dans le port de Caunos à l'époque impériale et le commerce de la poix et de la résine (cf. G.E.BEAN, JHS 74 [1954], p.97-105, n.38). 


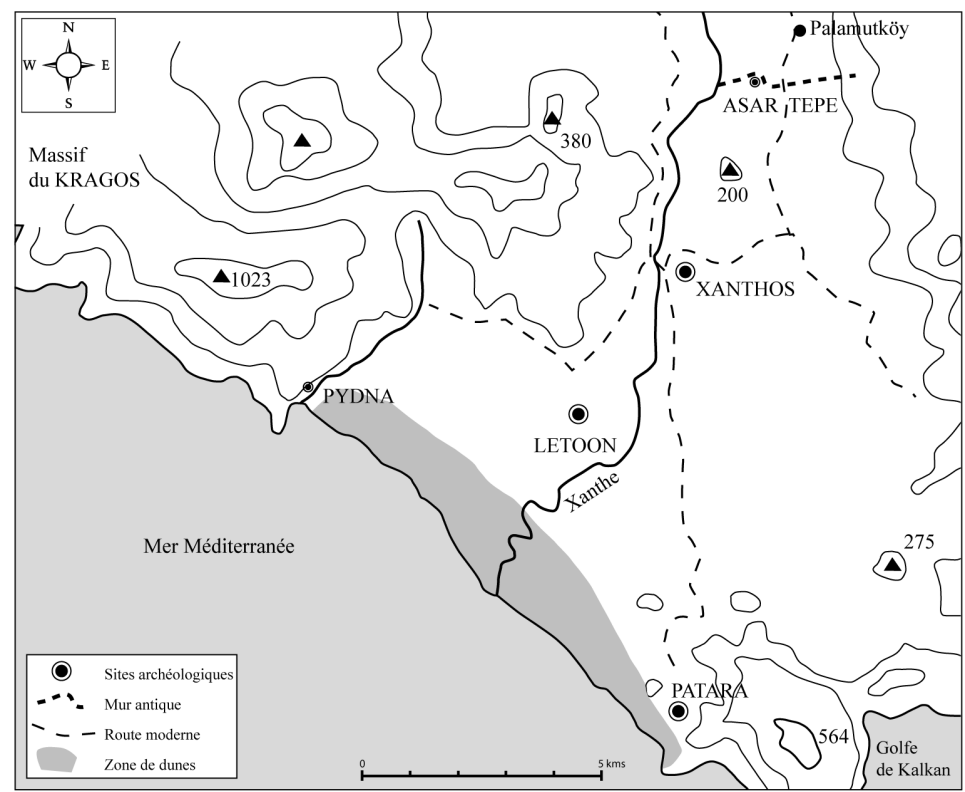

Fig. 1 - Carte de localisation du Létôon de Xanthos en Lycie, d'après P. Lebouteiller in J. Des CourtiLs, Guide de Xanthos et du Létôon, Istanbul (2003).

éventuellement les îles Chélidoniennes (cap Kélidonia) ${ }^{5}$, à la côte occidentale de Chypre, la péninsule d'Akamas et Paphos en particulier (voir la carte présentée Fig. 15) ${ }^{6}$.

Hormis les mentions sur la persistance des échanges nécessaires au commerce du cuivre ${ }^{7}$, bien peu de témoignages écrits sur les échanges concernant Chypre sont connus pour l'époque romaine. L'examen des vestiges mobiliers des deux régions, représentées l'une par le Letôon de Xanthos, l'autre par les contextes

5. Aucun port dans ces îles n'est attesté; et si l'on peut supposer que l'une d'elle permettait une escale (Strabon, Géographie, 14, 3, 8: «l'une d'elles possède un bon mouillage»), il est peu probable qu'un centre d'échanges y fût établi (Pomponius Mela, La description de la terre, 2, 7: «On appelle Chélidoniennes, celles qui font face au promontoire du Taurus, et dont l'approche est si dangereuse aux navigateurs». Pline, Hist. Nat., 5, 35: «les trois îles Chélidoniennes, funestes aux navigateurs»). Voir également sur ce point ARNAUD 2005, p.210, à propos de ces îles de rochers.

6. Arnaud 2005, p.211. L'auteur mentionne «les alignements remarquables» qui caractérisent cette région selon les géographes de l'Antiquité. Voir également la carte p.212. Plus récemment, ARNAUd 2011.

7. Charlesworth 1961, p. 87-88. 

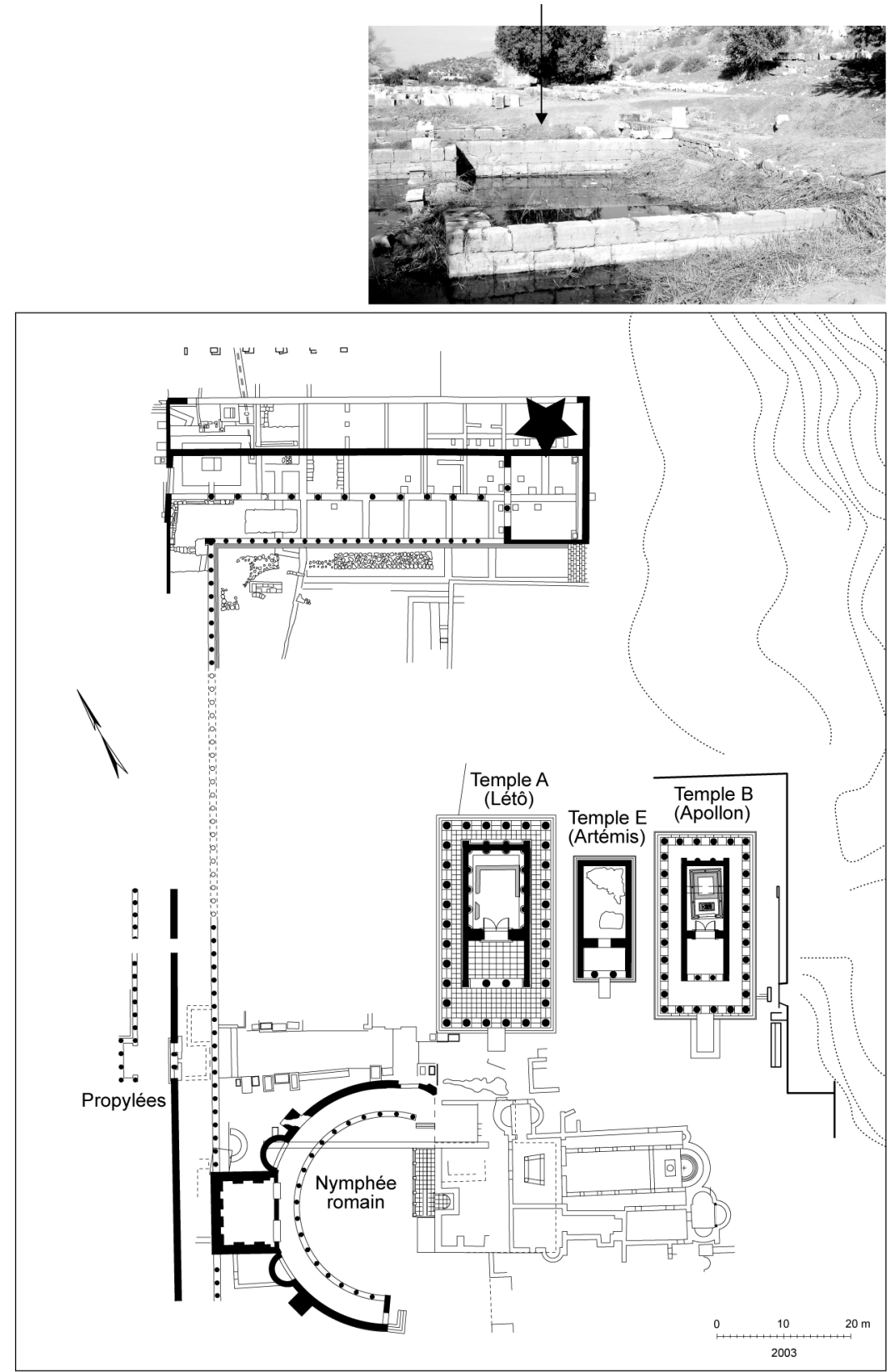

Fig.2 - Plan du Létôon de Xanthos: localisation de l'ensemble étudié. Plan D. Laroche. Cliché S. Lemaître. 
domestiques de $\operatorname{Paphos}^{8}$, permet de préciser leurs orientations économiques et culturelles.

\section{Contexte archéologique}

En 1972, l'exploration de la zone des portiques nord du Létôon de Xanthos, entreprise par H. Metzger ${ }^{9}$, mettait au jour une couche très riche en matériel et localisée entre le mur de péribole et le mur de fond de la salle, dite «impériale ${ }^{10}$ (Fig.2).

Le lieu de découverte de ce remblai, aux abords immédiats de l'espace sacré, permet d'établir un lien entre les artefacts livrés par la couche archéologique et la vie du sanctuaire ${ }^{11}$. Les conditions d'accès au mobilier dont l'étude avait débuté dès $2003^{12}$ ont été particulièrement chaotiques, ce qui explique en partie le laps de temps entre l'étude et la publication. Cet ensemble comprend aux côtés des mobiliers céramiques de nombreux fragments de verre ${ }^{13}$, une ou deux monnaies ${ }^{14}$ ainsi que des restes fauniques.

Le lot est composé d'un peu plus de 5800 tessons de céramiques. Au sein du remblai NE/D-E17, les amphores constituent $48 \%$ du nombre de restes, contre $14 \%$ pour les céramiques fines, la dernière partie étant constituée par les céramiques communes de cuisine et de rares tessons de lampes à huile.

Du point de vue chronologique, la présence d'éléments relativement récents, notamment de quelques fragments d'amphores et de verres appartenant aux grandes séries de l'Antiquité tardive, conduit à considérer que l'ensemble des mobiliers était associé à un remblai mobilisé dans le courant $\mathrm{du}_{\mathrm{IV}}^{\mathrm{e}} \mathrm{ou} \mathrm{V}^{\mathrm{e}}$ siècle apr. J.-C. Celui-ci a dû être mis en place afin d'exhausser le niveau de circulation dans cette partie du site peut-être à la suite d'une remontée du niveau de la nappe phréatique,

8. Outre les données publiées par J. Hayes (HAYES 1991), les observations développées ici se fondent sur l'examen d'ensembles céramiques en cours d'étude issus des fouilles françaises de la colline de Fabrika à Paphos (dir. Cl. Balandier, Université d'Avignon).

9. MetzGer 1978. Ces caisses portaient toutes les mêmes indications de provenance: Letôon 1972 NE/D-E 17.

10. Metzger 1978, p.802.

11. Ces mobiliers doivent faire l'objet d'une publication sous la forme d'un ouvrage dans la collection Fouilles de Xanthos.

12. LEMAîTRE et al. 2004.

13. L'étude en a été confiée à S. Baybo.

14. D'après l'inventaire des objets de Chr. Le Roy, il s'agit de bronzes illisibles. 
un problème auquel les personnes fréquentant le site ont été confrontées dès les premières phases d'aménagement ${ }^{15}$.

Il semble, à ce stade de l'étude, que la plus grande partie des mobiliers composant l'assemblage puisse être datée à partir du milieu du $\mathrm{I}^{\text {er }}$ siècle apr. J.-C., au moment où la Lycie, fidèle alliée de Rome suite à la restitution par cette dernière de son indépendance en 166 av. J.-C., devient province romaine (48 apr. J.-C.) et voit se développer une activité économique intégrée à l'Empire ${ }^{16}$.

\section{Les imitations de céramiques fines importées au Létôon et leurs modèles chypriotes et rhodiens (S.Élaigne)}

La plupart des céramiques fines importées ont pu être rattachées à des centres de production orientaux majeurs de l'Orient méditerranéen des époques hellénistique et romaine ${ }^{17}$. Parmi ces importations, on a pu attribuer une origine chypriote à l'une d'entre elles et identifier une production d'imitation, témoignant d'une assimilation culturelle. Cette contribution nous donne donc l'occasion de faire le point sur les productions chypriotes de céramiques fines hellénistiques et romaines plus ou moins précisément définies.

Tout d'abord, les sigillées chypriotes ${ }^{18}$ ont pu être facilement déterminées étant donné l'existence d'une documentation précise et fiable concernant leur identification et leur répertoire typologique ${ }^{19}$. Il s'agit d'une production à pâte non calcaire de coloration orangée et à vernis rouge orangé de bonne qualité (brillant ou luisant, adhérent et imperméable). Cette fabrique reste mal localisée dans l'île, les structures de production n'ayant pas été découvertes. Toutefois, différentes séries d'analyses physico-chimiques permettent de proposer la côte nord comme hypothèse d' origine ${ }^{20}$,

15. Voir à ce propos la zone des portiques nord dans DAVESNE 2000.

16. Des Courtils 2007, p. 18-19.

17. Voir la contribution de S. Élaigne dans Lemaître et al. 2004, p.329-331, «Le mobilier céramique d'un remblai romain dans le sanctuaire du Letôon».

18. Pour un bilan des caractéristiques techniques et archéométriques de cette production, cf. ÉLAIGNE 2012, p.158-159.

19. Hayes 1985 pour les références typologiques. MeYZa 1995, p. 179-202; Lund 1997, p.201-215.

20. SCHNEIDER 2000, p.533 sur les ES D: «the composition is not related to ophiolitic region in Cyprus ». Picon, Bondé 2002, p. 14. Pour préciser ces données, se reporter à la carte géologique simplifiée de Chypre (http://www.cyprusgeology.org/english/ images/2_F1.htm). 

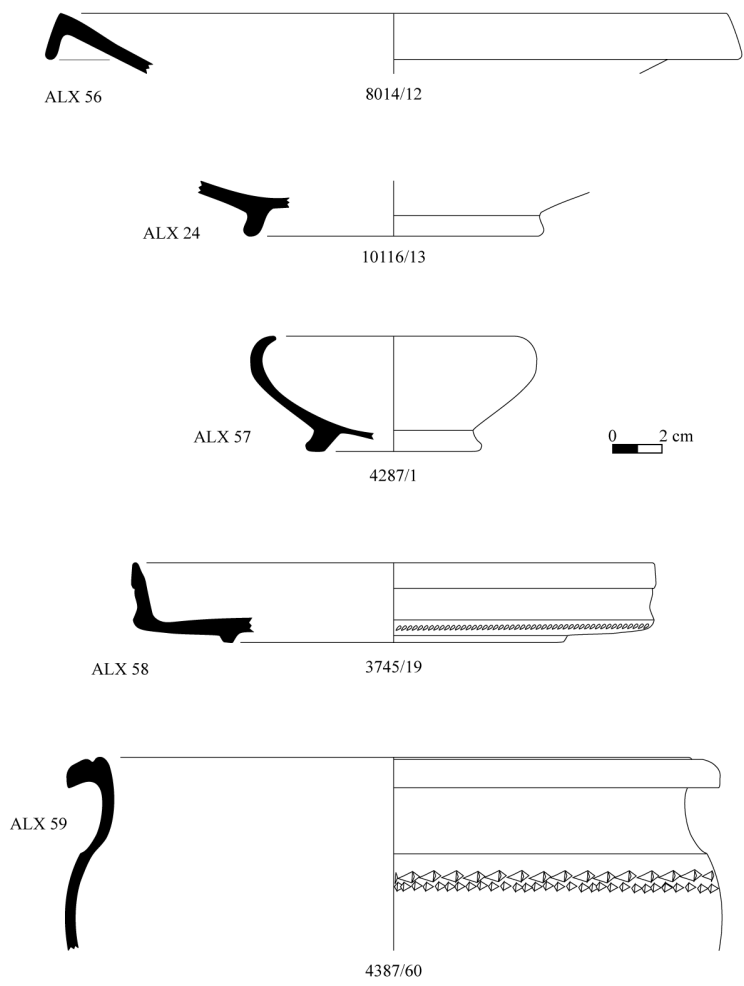

Fig.3- Sigillées présumées chypriotes trouvées à Alexandrie et analysées par méthode physico-chimique. Dessins S. Élaigne.

et éventuellement la région de $\mathrm{Soli}^{21}$. En outre, les investigations archéométriques ont montré qu'une production antérieure à ces sigillées romaines, employant les mêmes argiles non calcaires, puisque ces deux productions possèdent de fortes affinités de compositions chimiques, avait existé dès le $\mathrm{III}^{\mathrm{e}}$ siècle av. J.-C. ${ }^{22}$. Certains de ces prédécesseurs hellénistiques appartiennent à l'une des séries chypriotes désignées par J.Hayes comme «Colour-Coated ware ${ }^{23}$. D'ailleurs, lors de l'étude

21. MeYZa 2002, p.26 et note 20.

22. Picon, Blondé 2002, p. 15 sur le groupe D. MeYZa 2002, p. 26, sur les prédécesseurs à vernis bicolore de la sigillée chypriote.

23. MeyZa 2002,p.26. Les observations de l'auteur concernant la série qui porte un décor peint sur le vernis bicolore méritent précision: en effet, cette autre série possède des caractéristiques macroscopiques proches des prédécesseurs hellénistiques des sigillées chypriotes, mais il semble vraiment s'agir d'une production différente, qui appartiendrait au groupe A (cf. infra note 28), rattachée à la région de Kition suite 
des importations de céramiques fines à Alexandrie ${ }^{24}$, quelques échantillons à pâte argileuse rouge orangée (c'est-à-dire non calcaire) présumés chypriotes ${ }^{25}$, mais dont la typologie n'était pas forcément significative (Fig.3), ont été comparés par analyse physico-chimique au groupe de référence des sigillées chypriotes du Laboratoire de Berlin ${ }^{26}$. Cette confrontation conforte l'appartenance d'un échantillon ancien au groupe chypriote non calcaire (c'est-à-dire au groupe D) ${ }^{27}$. Ainsi, la production de sigillées chypriotes pourrait avoir été précédée par une production hellénistique utilisant des argiles identiques ou très proches dans la même zone géographique.

Les importations de sigillées chypriotes sur la côte sud-anatolienne et en particulier sur la côte lycienne sont attestées dans d'autres sites ${ }^{28}$. Dans l'assemblage du Letôon, les importations de sigillées chypriotes représentent environ $5 \%$ du nombre minimum de vases intégrant la catégorie des céramiques fines (Fig.4 et 5). Les formes impériales sont datées du milieu du $\mathrm{I}^{\mathrm{er}}$ au milieu du $\mathrm{II}^{\mathrm{e}}$ siècle (plats de type P11 et P12 et bol P40), mais elles sont accompagnées de formes un peu plus anciennes, caractéristiques du $\mathrm{I}^{\mathrm{er}}$ siècle (plats P4B, P5 et P10 et bols P22A et P28). Le répertoire enregistré est diversifié, combinant plats, bols et lagynoi. Les plats de la première moitié du ir siècle, P4B (F129), P5 (F279), P8 (F94) et P10 (F131), sont représentés par un seul exemplaire, et sont associés à un pied de P1 (F145) hellénistique, donc résiduel. Les types de plats et de bols, produits à partir de la seconde moitié du $\mathrm{I}^{\mathrm{er}}$ siècle, ont été répertoriés au moins en deux exemplaires. C'est le cas des plats P11 (F130, F133, F144), et P12 (F132, F134, F135) ainsi que des bols P22A (F138, F139, F151), P28 (F136, F137), et P40 (F143-152, F148). Bien que les fragments de lagynoi soient malaisément identifiables, on a cru reconnaître le type X51 (F146 et F150) et le type X56 (F140), deux fonds de vases fermés (peut-être X52) restant indéterminés (F84, F149).

Une autre production chypriote impériale, récemment documentée à partir de l'étude des céramiques fines de Kition, propose un répertoire qui reproduit

aux travaux de S. Marquié (MARQuí 2004). Elle précède la série d'imitations locales de sigillées (Élaigne 2007, p.120-121, § 2, fig. 19).

24. Cf. Élaigne 2012.

25. Ce groupe, comprenant toutes les productions à pâte foncée et revêtement rouge désignées comme «chypriotes », a été déterminé par les travaux de G. Schneider : cf. MEYZa 1995, p. 185, tab. 1.

26. SCHNEIDER 2000, p. 533.

27. Élaigne 2002, p.162 et 170, fig.8. Picon, Blondé 2002, p.14-15, fig.2. Élaigne 2012, p. 159: plat à paroi oblique et lèvre tombante ALX 56 (fig. 53, 8014/12) dans un contexte de la seconde moitié du II ${ }^{\mathrm{e}}$ siècle av. J.-C.

28. Notamment à Limyra: Yener-Marksteiner 2007, p.249-250 et Taf. 17, B21 à B24. 

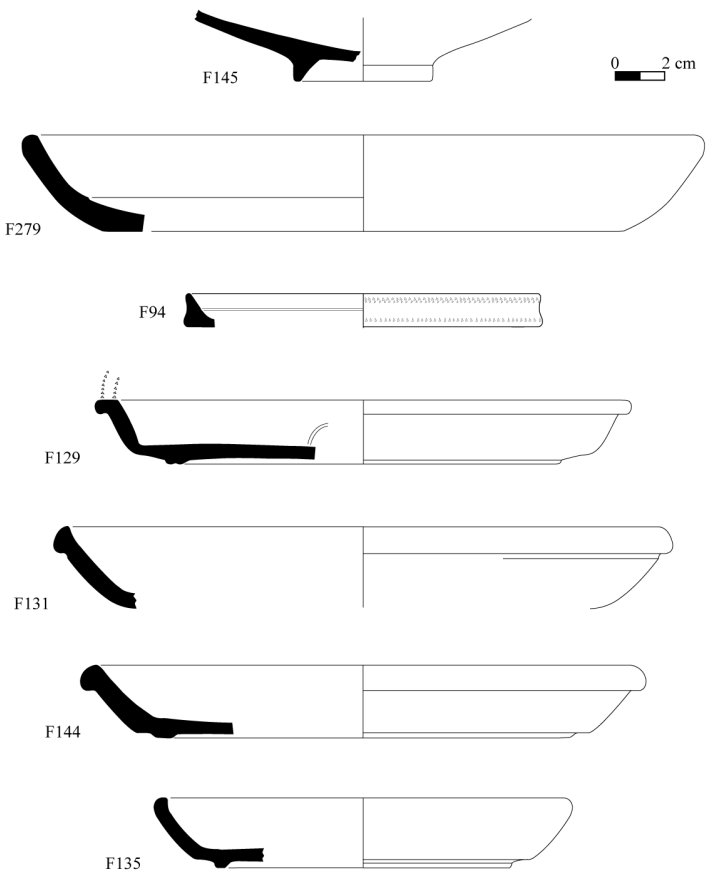

Fig.4- Létôon, NE/D-E17 : sigillées chypriotes. Dessins S. Élaigne.

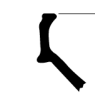

F136

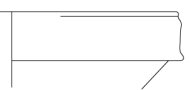

$0 \quad \operatorname{ram}^{2 \mathrm{~cm}}$

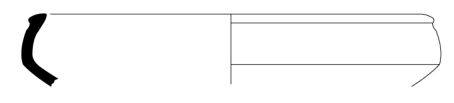

F138
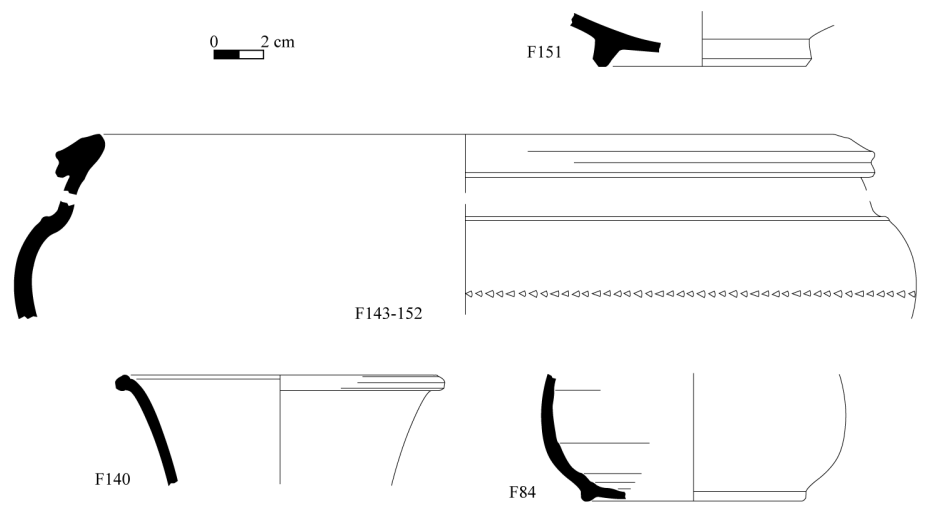

Fig.5 - Létôon, NE/D-E17 : sigillées chypriotes. Dessins S. Élaigne. 
certaines formes du répertoire des sigillées chypriotes ${ }^{29}$. Il s'agit d'une production homogène présentant une pâte argileuse de couleur variant du rose-orangé au brunâtre selon les températures de cuisson, avec des marbrures dans certains cas, et comprenant, outre de nombreux pores, diverses inclusions visibles, notamment des nodules rouges (oxydes de fer) et blancs (calcite), ces derniers se modifiant en efflorescences en cas de cuisson poussée. Le vernis bien adhérent affiche aussi des colorations très variables rouge-orangé plus ou moins brillant à brun luisant. Cette production d'époque romaine à pâte moyennement calcaire (comprenant en moyenne 9 à $12 \%$ de $\mathrm{CaO})^{30}$ imite essentiellement les formes $\mathrm{P} 11$ et P40 des sigillées. On suppose donc qu'il puisse s'agir d'une production de la région de Kition, bien qu'il soit encore nécessaire de vérifier les éventuelles similitudes de composition de ces productions avec des argiles locales. Les formes reproduites, vraisemblablement localement à Kition, ne le sont qu'à partir du début du II siècle, moment où leurs modèles sont introduits ${ }^{31}$.

En outre, les investigations physico-chimiques réalisées au Laboratoire de céramologie de Lyon, portant d'une part sur la série impériale de Kition ${ }^{32}$, d'autre part sur certaines céramiques hellénistiques à vernis noir et/ou rouge prélevées dans des contextes d'habitat de Kition, Amathonte et Paphos, et rassemblées dans le groupe $\mathrm{A}^{33}$, font apparaître que les compositions des deux ensembles appartiennent à une même origine. Évidemment, l'observation macroscopique des caractéristiques techniques des pâtes argileuses des deux ensembles confirme leur similitude. Cette production hellénistique, précédant la production impériale d'imitation des sigillées chypriotes, supposée provenir de Chypre orientale et vraisemblablement de la région de Kition, semble avoir été diffusée à l'échelle régionale, et essentiellement vers l'Est, sur la côte levantine, comme certaines observations réalisées à partir du mobilier de Beyrouth ${ }^{34}$ et de Tel Dor et Tell

29. Cf. les travaux de S. Marquié dans le cadre de sa thèse (MARquí́ 2004), p. 176-177.

30. MARQuí́ 2004, Annexe 4.2.1.

31. MARQuí́ 2002, p. 294: «l'ES A (...) est peu à peu remplacée par la $C S$ (...) au tout début du II à Kition».

32. MARQUiÉ 2004, Annexe 4.2.1. sur le «groupe calcaire».

33. Picon, Blondé, p. 15 et p. 20 , tableau 1, groupe A.

34. Une série de vases hellénistiques, à vernis bicolore, comprenant des bols echinus et des plats de type Jehasse 23/36 (petit plat à cupule et rebord) répertoriés à Beyrouth et présentant une pâte argileuse macroscopiquement similaire, semble devoir être rattachée à ce même groupe : cf. ÉLAIGNE 2007, p.120-121, § 2 et fig. 3 et 4 pour le répertoire. Cf. aussi ÉlAIGNE 2012, p.148, n.699, sur les investigations physicochimiques. 
Keisan ${ }^{35}$ en témoignent ${ }^{36}$. En revanche, aucune mention de diffusion pour les productions impériales d'imitation de ces sigillées chypriotes n'est connue à ce jour.

Un phénomène d'imitation du répertoire des sigillées chypriotes comparable a été déterminé lors de l'étude du corpus du Létôon, en premier lieu à partir de vases dont la typologie rejoint celle des sigillées chypriotes. Ce groupe fait, par conséquent, état d'une production lycienne impériale d'imitation des importations chypriotes. La pâte argileuse employée localement est très calcaire, présentant une coloration rosée à beige, qui peut s'éclaircir en surface jusqu' au blanchâtre ${ }^{37}$, et au toucher savonneux, assez éloignée de l'aspect macroscopique du modèle chypriote non calcaire, et par là-même facilement dissociable. Le vernis est médiocre: mince, le plus souvent mat, de couleur orangée. Par ailleurs, les types identifiés sont plus ou moins fidèles aux modèles à pâte non calcaire rouge-orangé appartenant à la catégorie des sigillées chypriotes. Ce groupe rassemble, comme les sigillées chypriotes, environ $5 \%$ des céramiques fines de l'assemblage.

Cette production calcaire impériale à engobe orangé se situe, du point de vue technologique, dans le continuum des céramiques fines produites localement ${ }^{38}$, tandis que son répertoire de formes s'inspire des importations régionales et en particulier des sigillées chypriotes (Fig.6). Elle est principalement illustré au Létôon par des bols de type P37B et P40. En outre, les plats P4B, P10, P11 et les lagynoi X58 et X59 (X51 et X52 avec moins de certitude) sont aussi présents dans notre assemblage. Les exemplaires reproduisant le plus fidèlement les sigillées chypriotes concernent les types canoniques P4B, décorés de guillochis sur le rebord (3 vases dont F280 et F297), la coupe P37B (6 vases au total de ce type dont F274 et F291), le bol P31A (F347) et les bols de grand module P30 (F275) et P40 (6 vases en tout dont F284). En outre, d'autres formes plus éloignées du répertoire des sigillées chypriotes possèdent les mêmes caractéristiques techniques que le groupe chypriote à pâte calcaire (Fig.7).

35. MlynarczyK 2002, p. 120, et fig. 2, n² 28 à 42: des vases de Tell Keisan présentent de fortes similitudes techniques et morphologiques avec une production décrite à partir du mobilier des $\mathrm{IV}^{\mathrm{e}}$ et $\mathrm{III}^{\mathrm{e}}$ siècles de Kition et sont attribués avec réserve à Chypre orientale; il en va de même pour le cratère $\mathrm{n}^{\circ} 51$, fig. 3 , p. 121 , dont la présence est aussi attestée à Tell 'Arqa et Beyrouth (Élaigne 2007, fig.4, 800-10, et fig. 19).

36. Marquí 2002, p.294-295. Cette orientation de la diffusion concorde avec les observations de J.Lund qui s'appuie sur les itinéraires maritimes, les cargaisons destinées à la côte levantine empruntant la route sud qui faisait escale à Kition ou à Salamine.

37. LEMAîTRE 2007, p. 121-122 sur les caractéristiques techniques du premier groupe des céramiques fines engobées vraisemblablement produites localement aux époques archaïque et classique.

38. Les caractéristiques technologiques des productions locales de céramiques fines sont invariables depuis l'époque archaïque (cf. note précédente). 


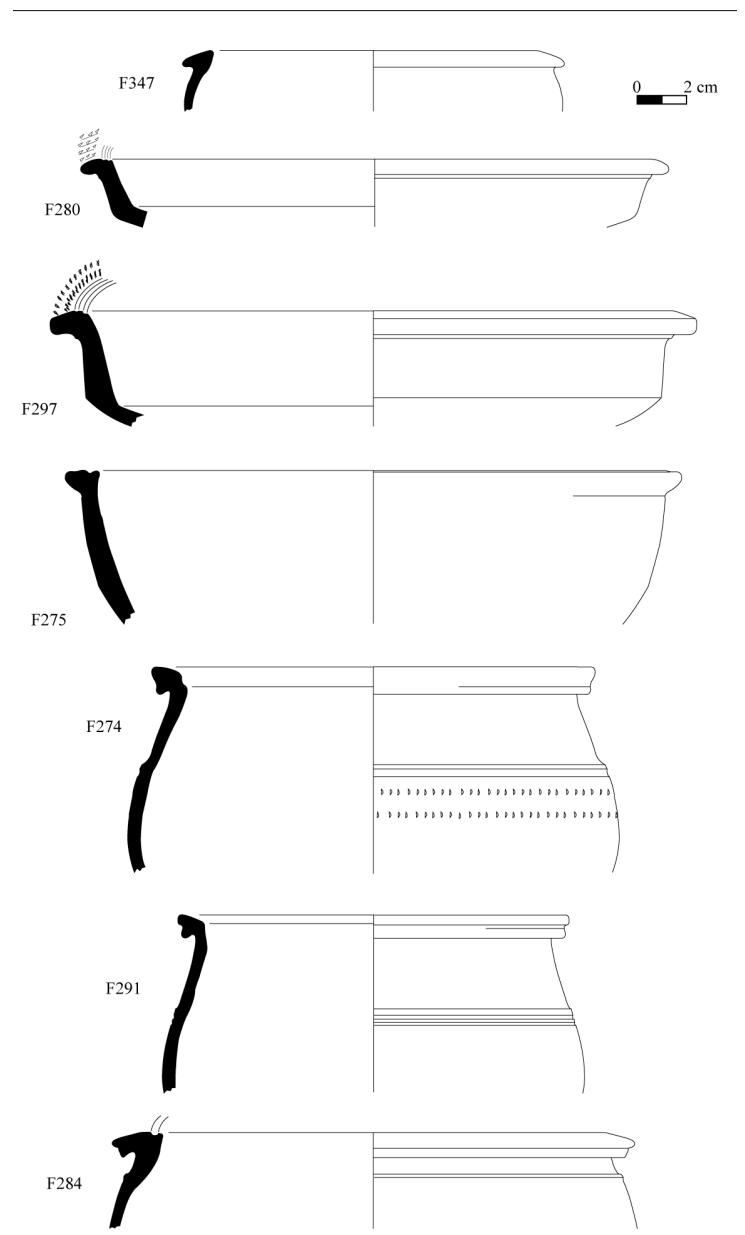

Fig.6- Létôon, NE/D-E17 : productions calcaires locales reproduisant le répertoire des sigillées chypriotes. Dessins S. Élaigne.

Celles-ci méritent quelques commentaires:

- F300 : il pourrait s'agir d'une variante «dégénérée » de la forme P37B des sigillées chypriotes.

- F348 : cette partie supérieure de vase trouve deux possibilités de parallèles (non exacts). Il peut s'agir d'une coupe à paroi oblique et lèvre ourlée, connue en quelques rares exemplaires de sigillée chypriote mais sans le bourrelet surmontant 


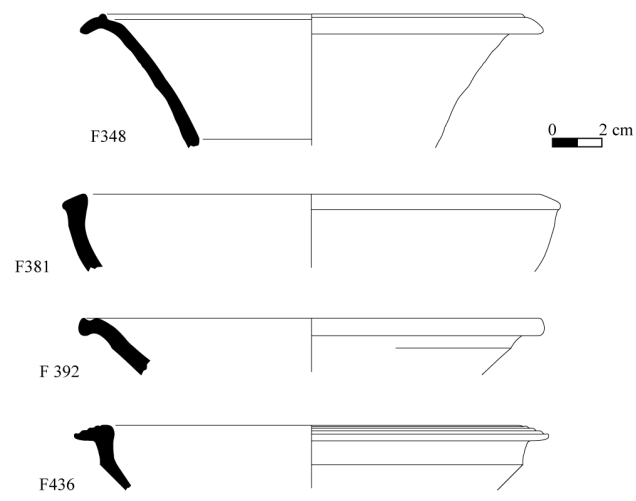

Fig. 7 - Létôon, NE/D-E17 : productions calcaires locales inspirées des formes de sigillées chypriotes. Dessins S. Élaigne.
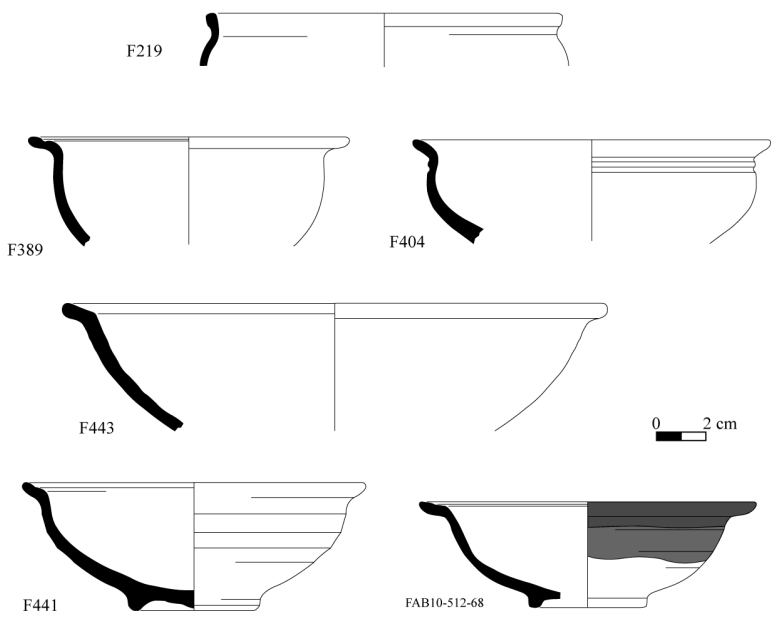

Fig. 8- Létôon, NE/D-E17: skyphos rhodien (F219), et bols hémisphériques à bord évasé en pâte calcaire locale avec leur modèle rhodien découvert à Paphos. Dessins S. Élaigne.

l'arête de la lèvre ${ }^{39}$; en outre, cette particularité de la lèvre se rencontre sur un bol caréné chypriote en pâte calcaire à Paphos ${ }^{40}$.

- F381 : cette forme est similaire au type P11 des sigillées chypriotes.

- F392: ce vase est proche du type P7 des sigillées chypriotes.

39. MeYZa 2002, p.29, fig. $3, \mathrm{n}^{\circ} 14$.

40. HAYES 1991, cf. bol caréné à lèvre ourlée et ressaut en pâte calcaire locale, p.28, fig. XV, $n^{\circ} 15$. 
- F436: ce type s'apparente au type P4 des sigillées chypriotes et à la forme tardive Hayes 7 des $«$ Cypriot Red Slip ware ${ }^{41}$.

Enfin, dans cette catégorie de productions à pâte très calcaire, donc très claire, il en existe une autre d'origine chypriote, très influencée par les productions rhodiennes ${ }^{42}$. Celle-ci avait été classée dans le vaste ensemble hellénistique dénommé «Cypriot Colour-Coated wares » par J.Hayes ${ }^{43}$ à partir des céramiques de contextes domestiques de Paphos ${ }^{44}$. Son répertoire d'époque hellénistique se décline essentiellement en bols echinus, plats à paroi oblique et olpai ${ }^{45}$. Il comprend aussi le skyphos à anses en accolade ${ }^{46}$, un type très particulier, facile à différencier (Fig.8: exemplaire du Létôon dont les anses manquent), et dont la présence est importante dans les régions voisines (Levant nord), alimentées par la route maritime qui relie Rhodes à Alexandrie ${ }^{47}$, longeant la côte orientale de Chypre, et particulièrement à Paphos, escale obligée. Inversement, ce type est plus rare à Amathonte et à Kition ${ }^{48}$, où le skyphos à anses-poucier prédomine. Paradoxalement, ces deux types de skyphos ne coexistent pas dans les assemblages, ni chronologiquement, ni géographiquement. Le skyphos à anses-poucier (forme Jehasse 43C), bien connu à Salamine, est rarement attesté à Paphos ${ }^{49}$, tandis qu'il existe en plusieurs exemplaires à Amathonte et à Kition ${ }^{50}$, ainsi que dans les sites du littoral levantin dans les ensembles du III $^{\mathrm{e}}$ siècle av. J.-C., notamment à

41. Williams 1989 , fig. $15, \mathrm{n}^{\circ} 199$.

42. Il s'agit du groupe «Colour-Coated ware A» défini dans HAYES 1991, p. 23-24.

43. HAYEs 1991, p. 26-29.

44. Il s'agit d'une série différente de celle citée par H. Meyza lorsqu'il évoque des parallèles technologiques et antérieurs aux sigillées chypriotes: cf. supra, note 22.

45. HAYes 1991, p.26.

46. Ce type de skyphos s'ajoute à un autre type, à anses-poucier, produit dans les deux autres fabriques chypriotes étudiées ici, de la partie orientale de l'île, c'est-à-dire celle de la région de Kition et celle, non calcaire, précédant les sigillées chypriotes.

47. Le skyphos à anses en accolade, comme les plats à paroi oblique et les bols echinus de la fabrique rhodienne, sont attestés à Alexandrie en quantité notable surtout au $\mathrm{II}^{\mathrm{e}}$ siècle av. J.-C.: ÉLAIGNE 2000, p. 99 à 112. ÉLAIGNE 2002, p.162: confirmation d'une origine rhodienne pour le groupe 4 (fig. 4 et 5) par comparaison des compositions chimiques avec celles d'amphores rhodiennes. ÉLAIGNE 2012, p. 137-147.

48. SALLEs 1993: un seul exemplaire, p. 198, n³22.

49. Papuci-Wladyka 1995, p. 250, pl. 4, nº 230.

50. Pour Amathonte, cf. Petit 1995, fig. 12; cf. aussi Burkhalter 1987, p. 376, nº 106 à 108. Pour Kition, cf. SALLeS 1993, p. 186, n²19, 220, 221; p. 197, n³21; p.202, $\mathrm{n}^{\circ} 360$ et $364 ;$ p. $266, \mathrm{n}^{\circ} 503$. 
Beyrouth ${ }^{51}$, Tell Keisan et Tel Dor ${ }^{52}$. Il faut aussi préciser que les exemplaires de Kition existent dans différentes fabriques ${ }^{53}$.

Des fragments épars de cette production chypriote hellénistique à pâte calcaire ont été identifiés dans l'assemblage du Létôon. Notons qu'il s'agit d'éléments résiduels, parmi lesquels on compte un pied annulaire (F296) et un col de lagynos (F382). D'autre part, les importations rhodiennes constituent $11 \%$ de l'ensemble des céramiques fines au sein du lot, tenant le troisième rang si l'on considère la quantité des importations de vaisselle selon leur provenance, derrière les ES A et les importations cnidiennes.

Ainsi, au moins trois productions hellénistiques, appartenant aux «Colour-Coated ware», sont aujourd'hui différenciées, même si la localisation géographique des ateliers reste bien floue. On a vu que les deux séries rattachées à la partie orientale de l'île et possédant une pâte rose ou orangée proposent un répertoire de formes correspondant plutôt au $\mathrm{III}^{\mathrm{e}}$ siècle av. J.-C. Celle qui présente une pâte très claire, dont l'origine chypriote semble devoir être recherchée dans la partie occidentale de l'île, reproduit, tant du point de vue technologique, que du point de vue morphologique, la production rhodienne de céramiques fines et ce, essentiellement entre le début du II $^{\mathrm{e}}$ et le milieu du i ${ }^{\mathrm{er}}$ siècle av. J.-C., moment où la production et la diffusion des sigillées chypriotes se développent. En effet, la série hellénistique inspirée des productions rhodiennes est de médiocre qualité (variabilité des colorations du vernis et imperméabilité aléatoire), ce qui ne constituait pas un obstacle à la diffusion des céramiques à cette période (de nombreuses autres productions étaient dans ce cas). La nécessité du contrôle strict des températures pour l'obtention d'un vernis de bonne qualité, en raison de la teneur en calcaire assez élevée, a pu contribuer à faire disparaître cette fabrique au profit d'une production à pâte peu calcaire, telle que celle des sigillées chypriotes.

Enfin, on est tenté d'établir une parenté typologique entre les nombreux bols hémisphériques à rebord ou bord évasé appartenant à la production locale à pâte calcaire de notre assemblage (Fig.8: F389, F404, F441, F443) et le type de bol similaire que l'on rencontre à Paphos dans la série des «Cypriot ColourCoated» à pâte calcaire ${ }^{54}$ provenant probablement d'un centre de production de

51. ÉLAigne 2007, p. 120-121 et fig.4.

52. MLynarczyK 2002, p. 120 , fig. 2 , n 38 et 39 .

53. SALLES 1993, p. 266, n 503 : «plusieurs anses à poucier faisaient également partie du remplissage (pâtes chypriotes et larnaciotes)». La remarque nous indique plusieurs aspects techniques différents de pâtes: la «pâte chypriote»(p.167) est soit fine, dure, homogène, beige rosé et vernis mat brun-rouge, soit plus tendre, rouge clair à rouge vif, vernis rougeâtre; la «pâte larnaciote» (p.168) est tendre à fines inclusions sableuses, beige-orangé à rouge-brun, vernis noir à brunâtre de mauvaise qualité.

54. Papuci-Wladyka 1995, p.272, pl. 4, nº 222 non commenté dans le texte; Hayes 1991, fig.XV, n 14, «Cypriot colour-coated», p.30. 
l'Ouest de l'île. Le répertoire hellénistique de cette série étant entièrement inspiré du répertoire rhodien comme on vient de le voir, la présence d'un bol du même type et d'origine assûrément rhodienne (Fabrika 2010 - 512/68, cf. Fig. 8) dans un contexte du ir siècle av. J.-C. à Paphos ${ }^{55}$, conforte l'existence de liens privilégiés entre la Lycie, la côte occidentale de Chypre et Rhodes à l'époque gréco-romaine. Ces relations se manifestent par l'intégration, en Lycie, et en particulier au Létôon, dans la vaisselle, de formes directement issues des manières de table chypriotes et rhodiennes. L'existence de cette communauté culturelle n'est sans doute pas étrangère à l'intensité des relations économiques internes à cette région, qui ont contribué à désenclaver la côte lycienne aux époques hellénistique et romaine.

\section{Du vin d'origine chypriote consommé au Létôon? (S. Lemaître)}

Issus du même ensemble que les céramiques fines qui viennent d'être présentées, des tessons d'amphores possédant des caractéristiques morphologiques communes conduisent à évoquer la présence et la consommation, dans le sanctuaire, de vin (?) peut-être originaire de Chypre, et d'une manière générale les relations, dont la nature reste encore à préciser, entre le Létôon et plus globalement la Lycie, la Cilicie et Chypre.

Le remblai fouillé dans les carrés NE/D-E 17 comptait un peu plus de 2800 tessons d'amphores, soit $48 \%$ du nombre total de restes de céramiques, à partir desquels on a pu dénombrer 106 individus en NMI ${ }^{56}$ et 440 en NTI ${ }^{57}$.

La grande majorité des amphores de l'assemblage correspond à des productions que l'on peut placer entre la deuxième moitié du $\mathrm{I}^{\text {er }}$ siècle et la première moitié du $\mathrm{II}^{\mathrm{e}}$ siècle apr. J.-C. Il faut toutefois noter la présence de quelques amphores datées des $\mathrm{II}^{\mathrm{e}}$ et $\mathrm{I}^{\mathrm{er}}$ siècles av. J.-C. en position résiduelle (amphores italiques Dressel 1 et Lamboglia 2 et une partie des amphores rhodiennes, ainsi que plusieurs amphores de Cnide estampillées datées des $\mathrm{III}^{\mathrm{e}}$-II ${ }^{\mathrm{e}}$ siècles av. J.-C.). Cinq tessons, nettement plus tardifs, d'époque proto-byzantine, doivent être interprétés comme des intrusions des niveaux supérieurs.

55. Il s'agit d'un remblai préalable à l'installation d'un habitat d'époque sur la colline de Fabrika (fouille dirigée par $\mathrm{Cl}$. Balandier, Université d'Avignon).

56. Nombre Minimum d'Individus calculé à partir des fragments de bords (après collage). Ce type de comptage permet de préciser la part de chaque région dans l'approvisionnement, sur le site et à un moment donné.

57. Nombre Typologique d'Individus (NTI) calculé d'après tous les fragments identifiables (après collage). Il met en lumière certains types d'amphores présents en faibles quantités et qui n'apparaissent pas dans les comptages en NMI. 


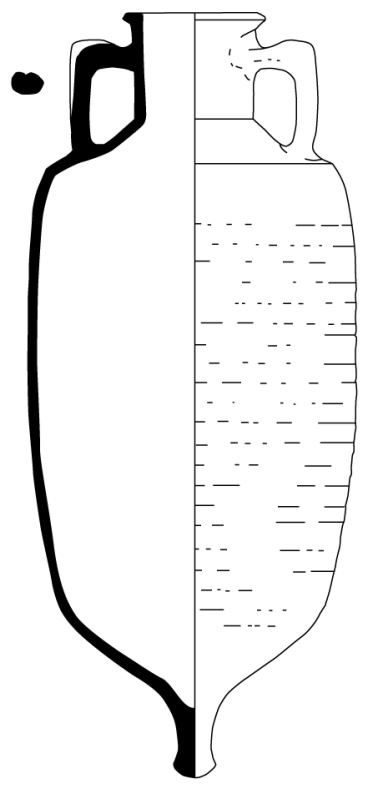

Fig.9- Morphologie générale de l'amphore Agora G199 /

Schöne-Mau XXVII-XXVIII, d'après PANELLA 1973, p.631, 34. Echelle 1/10.

Les individus répertoriés se répartissent entre une trentaine de types, importés de la région proche (côte cilicienne), d'Asie Mineure, de la côte levantine ou même du bassin occidental de la Méditerranée ${ }^{58}$.

Parmi les amphores, 18 fragments identifiables appartiennent à des amphores de type Agora G199. Individualisée en premier lieu dans les fouilles des cités vésuviennes, cette amphore porte le numéro XXVII-XXVIII de la typologie des amphores de Pompéi ${ }^{59}$. Elle apparaît également parmi le matériel amphorique des fouilles américaines de l'Agora à Athènes dans le niveau supérieur (III) du comblement d'une citerne, daté de la fin du $\mathrm{I}^{\mathrm{er}}$ et du début du $\mathrm{II}^{\mathrm{e}}$ siècle apr. J.-C. ${ }^{60}$. Nous avons adopté l'appellation de la publication du matériel des fouilles américaines de l'Agora ${ }^{61}$. Dans la littérature archéologique anglosaxonne ce conteneur est souvent désigné sous la terminologie «pinched handle amphora ${ }^{62}$.

Cette amphore a une forme générale cylindrique. La lèvre, en bourrelet plus ou moins saillant est associée à un col cylindrique assez large et court (Fig.9). La liaison, entre le col et l'épaule oblique, lisse et carénée, est souvent soulignée par un sillon ou une nervure. La transition avec la panse garnie de cannelures aplaties et peu accentuées s'effectue brutalement. Celle-ci se termine par une pointe, éventuellement pourvue d'un bouton ou torsadée. La principale caractéristique morphologique du type réside dans l'habitude qu'ont pris les potiers de pincer le

58. Sur la composition de l'assemblages des amphores, voir Lemaître 2006, p.397, fig. 10 et p.398, fig.11. Nota Bene: une photographie ayant malheureusement disparu de la figure p.398, la légende ne correspond plus aux dessins figurant sur la planche. Ainsi, les numéros 6 à 9 sont-ils des conteneurs mono-ansés dont une partie est sans doute originaire d'Éphèse, le numéro 10 est manquant et les numéros 11 et 12 sont des amphores de type Agora G199. Le numéro 11 correspond à AM 31 du remblai NE/D-E 17, présenté ici fig. 12, tandis que le numéro 12 a été mis au jour à Limyra (Li 82. SO 3B. 44/45).

59. CILIV, forme XXVII-XXVIII.

60. RoBINSON 1959, p. 22.

61. RoBINSON 1959, p.43.

62. Lund 2000. 
sommet de l'anse. Elle présente une section pseudo-bifide ou ronde marquée par un sillon central. Assez courte, elle s'élève verticalement depuis la partie basse de l'épaule et rejoint le col nettement sous la lèvre.

Plutôt caractéristique des $\mathrm{I}^{\mathrm{er}}$ et $\mathrm{II}^{\mathrm{e}}$ siècles apr. J.-C., le type semble exister encore aux $\mathrm{III}^{\mathrm{e}}$ et $\mathrm{IV}^{\mathrm{e}}$ siècles de notre ère sous une forme évoluée, de capacité réduite ${ }^{63}$.

Ce conteneur a suscité depuis longtemps l'intérêt des archéologues, sans doute à cause de la découverte relativement précoce d'ateliers l'ayant fabriqué. Une origine dans le bassin oriental de la Méditerranée avait été envisagée très tôt par C. Panella eu égard aux inscriptions peintes en grec que portaient plusieurs exemplaires mis au jour à Pompéi et à certains traits morphologiques qu'elle décrivait comme appartenant à la sphère de production égéenne ${ }^{64}$. L'origine orientale et plus précisément sud-anatolienne de ce type d'amphore ne fait plus de doute aujourd'hui.

Nos connaissances ont en effet largement progressé grâce notamment à la publication d'un atelier ayant produit, entre autres choses, ce type de conteneur et localisé dans l'ancienne cité d'Anemurium, à proximité de l'actuelle Anamur sur la côte sud de la Turquie ${ }^{65}$. L'argile utilisée pour la fabrication des amphores est décrite comme: «medium-grained with a very gritty texture along the breaks. It is full of small white lime particles, small red and dark coloured grits, fine silver mica and silica flakes ${ }^{66}$. Les fragments présentent tantôt une couleur orange associée à un engobe mat blanc ou jaunâtre, tantôt une couleur rouge foncé lorsqu'ils sont plus cuits. La publication de plusieurs fragments provenant de l'atelier d'Anemurium montre une assez grande variété morphologique: lèvre en bourrelet plus ou moins saillant et diverses formes du pied (Fig. 10).

À la suite de cette découverte, différentes opérations de prospection en Cilicie Trachée ${ }^{67}$ ont conduit à la mise en lumière d'au moins trois ateliers supplémentaires ayant fabriqué des amphores Agora G199 «classiques» et/ou plus tardives, localisés sur la côte à l'ouest d'Anemurium, à Syedra, Biçkici et

63. Robinson 1959, L11 et M239. Une amphore, Agora L11, p.73-75 et Plate 16, provient du comblement inférieur d'un puits (Groupe L, Layer I) daté de la seconde moitié du $\mathrm{III}^{\mathrm{e}}$ siècle. Un autre exemplaire, Agora M239, p. 106 et Plate 28, appartient au Groupe M, Layer VII, daté du début du IV siècle apr. J.-C.

64. Panella 1973, p.475-476, à propos d'exemplaires de type Mau XXVII-XXVIII dont les inscriptions ont été publiées dans le volume IV du CIL, suivi par A. Tchernia dans TCHERNia 1986, p.241. Dans un seul cas, il est certain que le titulus pictus en grec appartient bien à une amphore de type Mau XXVII-XXVIII : CIL IV, 2, tav. II, n. XXVII, 6386.

65. Williams 1989.

66. Williams 1989, p.91.

67. Notamment le Rough Cilicia Archaeological Survey Project, voir RAUH 2004. 

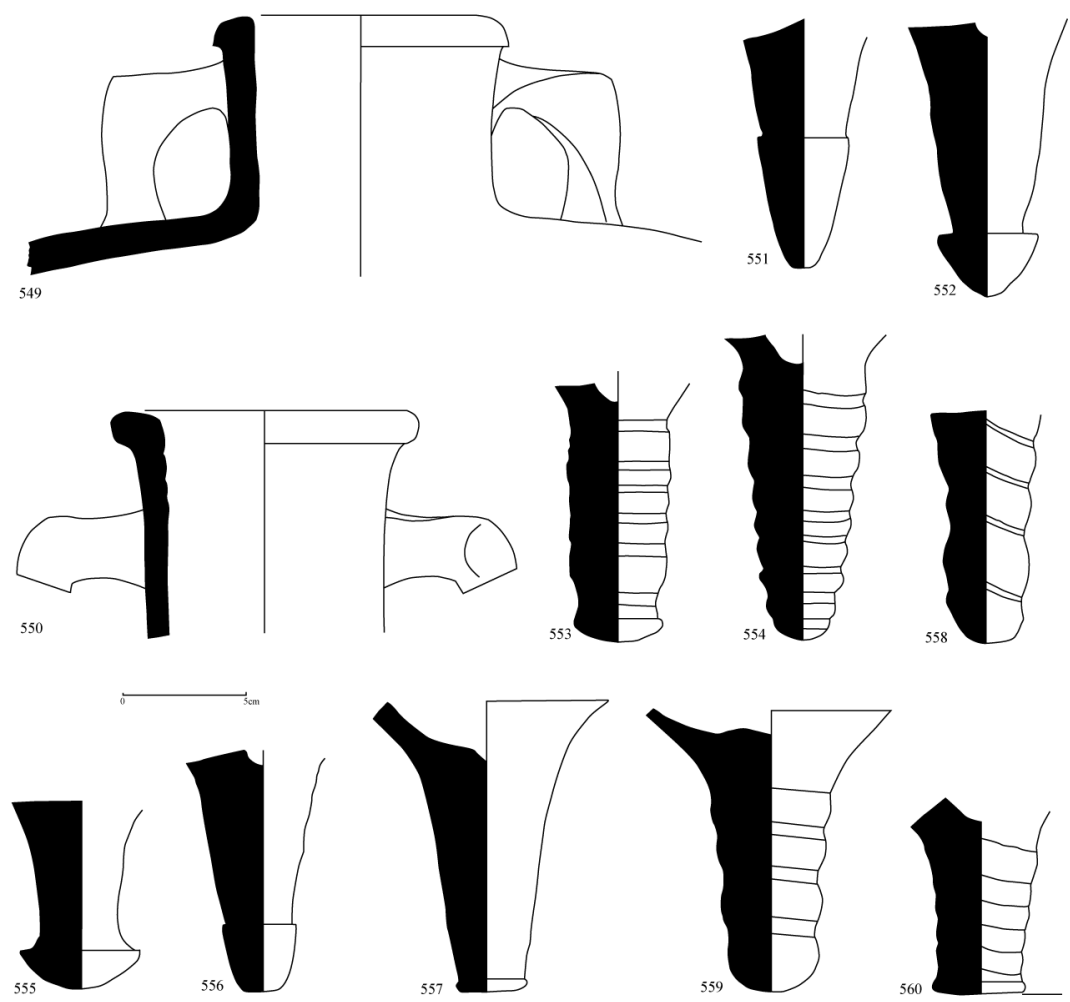

Fig.10- Fragments d'amphore Agora G199 provenant de l'atelier d'Anemurium, d'après Williams 1989, fig. 55. Echelle 1/4.

Antioche ad Cragum ${ }^{68}$. La pâte des productions de l'atelier de Syedra apparaît homogène: «... fine, redish yellow fabric with abundant, minute white inclusions and many tiny irregular voids; sparse angular or splintery white grains are also present and the surface is micaceous ${ }^{69}$.

À Biçkici, la pâte argileuse des imitations d'amphores de Cos et les Agora G199 présentent une pâte: «very fine light red usually with a buff surface. With a hand lens fresh breaks are micaceous and still very fine ${ }^{70}$.

Enfin, à Antioche ad Cragum, N. Rauh présente la "fabric" comme: «...fine grained, coarse breaking, medium soft. Many large red and white, some very

68. Voir sur ce point RaUH, SLANE 2000, Lund 2000, p. 569, RaUH 2004 et plus récemment Autret 2012, p. 255-256.

69. RauH 2004, p. 329.

70. Rauh 2004, p. 331 . 
small micaceous and white inclusions ${ }^{71}$. L'auteur précise que les analyses pétrographiques ont montré des similitudes entre cette pâte argileuse et celle de l'atelier repéré à Biçkici, s'expliquant peut-être par la proximité géographique des deux officines.

Les découvertes d'amphore Agora G199, nombreuses dans cette partie du Sud anatolien, font état d'une grande variété de pâtes argileuses, conduisant à supposer l'existence d'autres ateliers ayant produit ce type de conteneurs ${ }^{72}$.

Le même constat a été établi à Chypre, où ces amphores sont très largement répandues dans les zones littorales de l'ouest et du sud-ouest de l'île à Nea Paphos ${ }^{73}$. Sur la côte sud et dans l'est, les conteneurs à anse pincée sont attestés à Kourion, Dreamer's Bay, Amathonte, Aradippou, Kition, Salamis et Ayion Philon ${ }^{74}$. J.Lund précise qu'elles constituent plus de $20 \%$ des mobiliers amphoriques collectés à l'occasion d'un programme de prospection dans la région de l'Akamas, la pointe située au Nord-Ouest ${ }^{75}$. Une partie de ces découvertes correspond sans doute à des importations d'origine cilicienne, mais dès 1991, J.Hayes, en étudiant les mobiliers céramiques provenant d'habitats d'époque impériale à Paphos, avait distingué une pâte «...hard, pinkish-red..., fired brownish at the surface...» et une pâte «...yellowish buff..., slightly sandy and softer than the first one» ${ }^{76}$. Cette dernière, la mieux représentée au sein des ensembles de mobiliers étudiés à Chypre, est considérée comme locale, sans qu'aucune découverte d'ateliers ne vienne malheureusement pour le moment étayer cette hypothèse ${ }^{77}$.

Les amphores Agora G199 du lot du Létôon s'inscrivent parfaitement au sein des réflexions concernant ce type d'amphore. Elles appartiennent incontestablement aux séries du Haut-Empire et ont été façonnées dans des pâtes argileuses différentes. Les fragments se répartissent entre cinq groupes (Fig. 11).

Le groupe 1 rassemble sept fragments sur dix-huit. Il se caractérise par une pâte argileuse brun rouge foncé assez compacte avec d'assez nombreuses inclusions blanc mat de petite taille et un peu de mica fin. Appartiennent à ce groupe deux fragments de bord à lèvre en bourrelet aplati (Fig. 12, AM 31; AM 32 , non représenté), des anses de section arrondie marquée par un sillon central et pincée à leur sommet (Fig.12, AM 33, 34 et 35) et une épaule oblique et lisse

71. RAUH 2004, p. 332.

72. Idem.

73. HAYes 1991, p.91.

74. Lund 2000, p.567, complété par Autret 2012, p.262, Figure 17.7. L'amphore Agora G199 correspond au type 1 défini par l'auteur.

75. LuND 2000, p.567.

76. Cité par J.Lund (Lund 2000, p.569).

77. HaYes op.cit., Lund 2000, p.569, et Autret 2012. 


\begin{tabular}{|c|c|c|c|c|}
\hline Invent. & Fgt & NMI & NTI & Pâte \\
\hline AM 31 & bord & 1 & 1 & 1 \\
\hline AM 32 & bord & 1 & 1 & 1 \\
\hline AM 33 & anse & & 1 & 1 \\
\hline AM 33 & anse & & 1 & 1 \\
\hline AM 34 & anse & & 1 & 1 \\
\hline AM 35 & anse & & 1 & 2 \\
\hline AM 36 & fond & & 1 & 1 \\
\hline AM 37 & fond & & 1 & 1 \\
\hline AM 38 & épaule & & 1 & 3 \\
\hline AM 39 & bord et anse & 1 & 1 & 3 \\
\hline AM 40 & bord et anse & 1 & 1 & 3 \\
\hline AM 41 & anse & & 1 & 3 \\
\hline AM 42 & anse & & 1 & 4 \\
\hline AM 43 & anse & & 1 & 4 \\
\hline AM 44 & col & & 1 & 2 \\
\hline AM 45 & bord et anse & 1 & 1 & 2 \\
\hline AM 46 & anse & & 18 & 5 \\
\hline AM 47 & fond & & & 1 \\
\hline AM 90 & fond & & 5 & 1 \\
\hline & & 5 & 1 & \\
\hline
\end{tabular}

Pâte 1 : brun foncé micacée Pâte 3 : calcaire jaune

Pâte 5 : orange fine non micacée, surface crème
Pâte 2 : rose micacée Pâte 4 : calcaire rose plus épurée

Fig. 11 - Létôon, NE/D-E17 : tableau d'inventaire des amphores Agora G199 de l'ensemble NE/D-E 17.

(Fig.12, AM 38). Le fragment montre dans sa partie inférieure tout juste l'amorce des côtes qui garnissaient la panse. Enfin, un fond en pointe muni d'un petit bouton rappelle plusieurs des exemplaires publiés provenant du site d'Anemurium ${ }^{78}$.

Un deuxième groupe de pâte (2) compte quatre éléments : la partie inférieure d'une anse (Fig.13, AM 46), ainsi qu'un bord associé au sommet d'une anse (Fig.13, AM 45) d'un module plus petit que celui observé sur les autres fragments de bord. Cet exemplaire présente un col étroit différent de celui des formes plus tardives connues dans le matériel des fouilles de l'Agora à Athènes ${ }^{79}$. Il s'agit

78. Williams 1989, Fig. 55, 553, 557.

79. Robinson 1959, Pl. 28, M 239. 

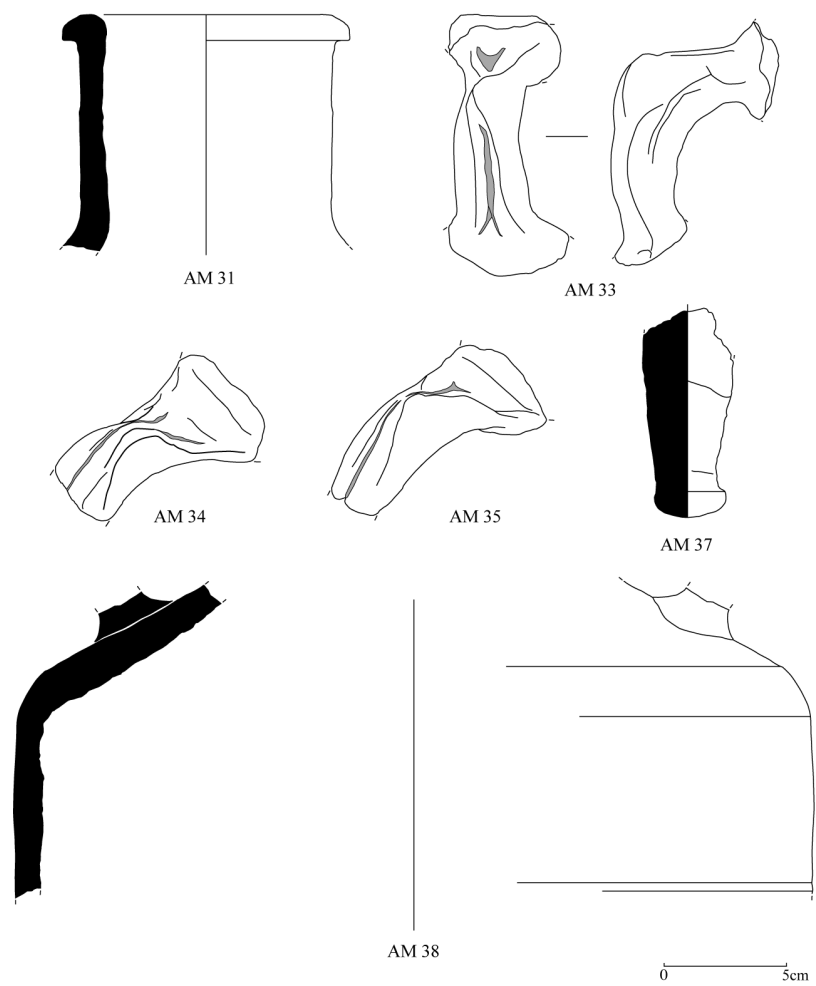

Fig.12 - Létôon, NE/D-E17: amphores Agora G199, groupe de pâte 1. Dessins S. Lemaître.

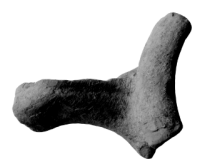

AM 45

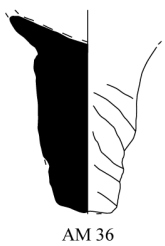

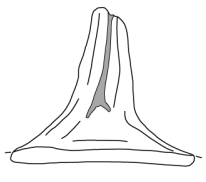

AM 46

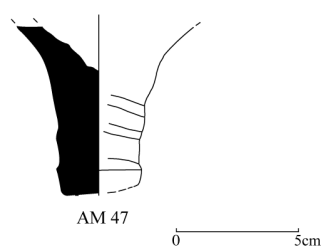

Fig.13- Létôon NE/D-E17 : amphores Agora G199, groupe de pâte 2. Dessins et photographie S. Lemaître. 


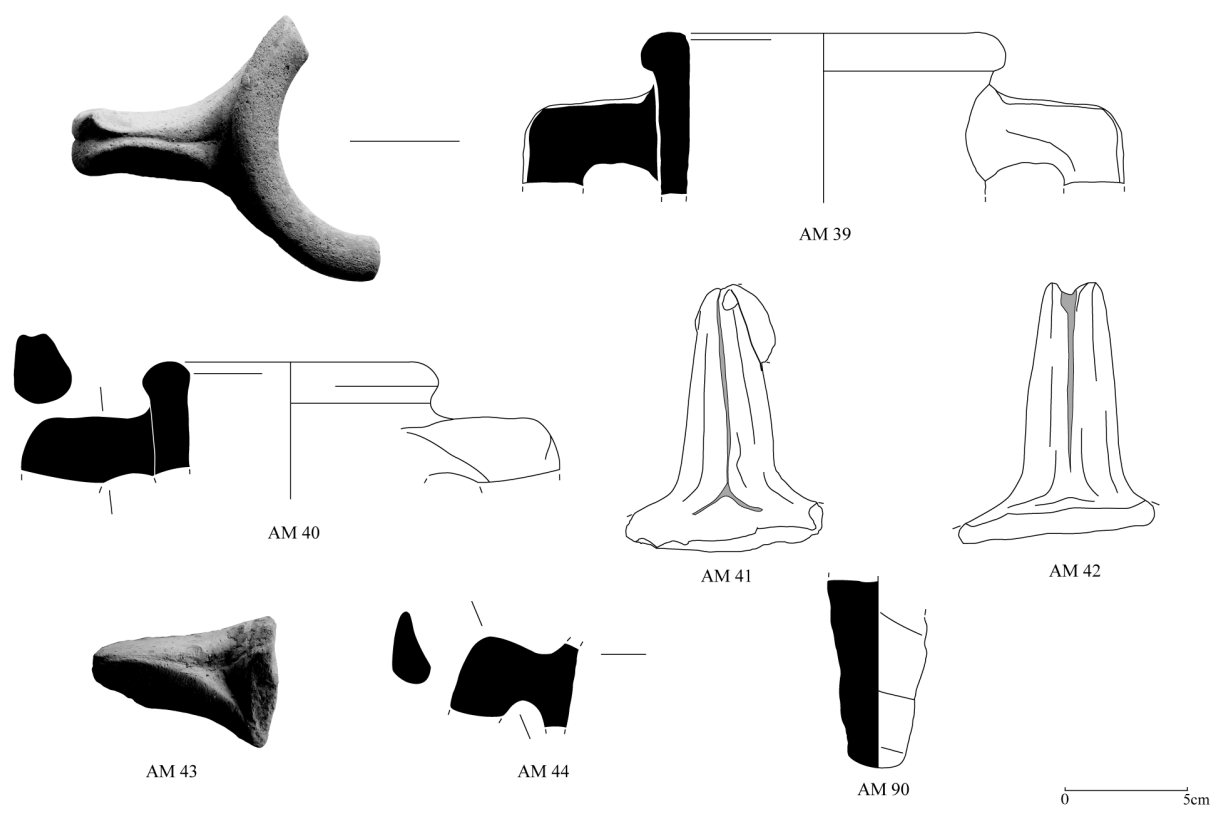

Fig. 14 - Létôon NE/D-E17: amphores Agora G199, groupes de pâte 3 à 5. Dessins et photographies S. Lemaître.

peut-être d'un demi-module ${ }^{80}$. Les fractions d'amphores, demi ou quart, sont un phénomène particulièrement développé en Méditerranée orientale à l'époque impériale, bien connu pour les productions crétoises notamment ${ }^{81}$. Deux fragments de fond, l'un conique torsadé (Fig.13, AM 36) et l'autre plutôt cylindrique muni d'un bouton (Fig.13, AM 47) complètent le groupe 2. Ces tessons ont en commun une pâte de couleur brun rose contenant des inclusions grises brillantes assez grossières et du mica blanc fin. La surface montre un engobe beige rosé partiellement conservé.

Quatre autres fragments d'amphores ont des caractéristiques macroscopiques communes (groupe 3). Il s'agit de deux bords et deux anses (Fig. 14, AM 39, 40, 41 et 42 ) au caractère assez massif. Tous ont une pâte calcaire de couleur beige jaune, non micacée. La surface présente un aspect rugueux lié aux nombreuses inclusions grises, rouges et translucides visibles dans l'argile.

Deux éléments supplémentaires (groupe 4) sont également associés à une pâte calcaire non micacée (Fig.14, AM 43,44). Ils ont été séparés du groupe

80. Une amphore Agora G199 à pâte rouge également de petit module est conservée au musée d'Antalya dans la vitrine consacrée aux découvertes de Perge.

81. Marangou-Lerat 1995, p.93-94. 
précédent car les inclusions y sont beaucoup moins abondantes dans une pâte de couleur rose et beige en surface.

Un dernier fragment de fond conique légèrement torsadé (Fig.14, AM 90) se trouve isolé dans un cinquième «groupe». Il a été façonné dans une argile proche de celle utilisée pour le groupe 1. Elle est orange et finement micacée.

$\mathrm{Si}$ une origine cilicienne peut raisonnablement être envisagée pour les amphores des groupes 1,2 et 5 , nous proposons d'assigner une origine chypriote aux fragments du groupe 3 en nous fondant sur les descriptions de fabric proposées par J. Hayes et J.Lund ${ }^{82}$ et sur la présence ou non de mica dans l'argile. La question est plus délicate pour le groupe de pâte 4. L'argile calcaire utilisée par les potiers et dépourvue de mica (en tous cas en quantité visible) paraît nettement différente de celle du groupe 3 . Elle se rapproche en fait de celle employée pour le façonnage des céramiques communes à pâte calcaire de production locales et/ ou régionales étudiées sur le site du Létôon ${ }^{83}$ mais également en Lycie orientale à Limyra ${ }^{84}$. Peut-être faut-il proposer d'étendre vers l'Ouest jusqu'en Lycie l'aire de production des conteneurs de type Agora G199? N. Rauh envisageait d'ailleurs l'existence d'autres ateliers de production que ceux mis au jour à l'heure actuelle ${ }^{85}$. Dans tous les cas, le recours à des analyses pétrographiques et/ou physico-chimiques doit être sérieusement projetée afin d'avancer sur les questions d'origine et de circulation des produits ${ }^{86}$, une démarche déjà appelée de leurs vœux par d'autres auteurs ${ }^{87}$.

Les conteneurs Agora G199 sont considérés comme des récipients destinés à la commercialisation du vin. L'hypothèse repose sur les informations livrées par les sources textuelles anciennes mentionnant la culture de la vigne dans les régions de production de ces amphores et sur l'observation de restes de résine

82. LuND 2000, p.569.

83. Les céramiques communes à pâte calcaire du dépotoir NE/D-E 17 ont été étudiées par C. Rocheron.

84. LEMAîTRE 2007, p. 215.

85. RAUH 2004, p. 332 .

86. C'est la démarche entreprise dans le cadre du programme franco-allemand Xanthiaca (2011-2013) financé par l'ANR et en particulier au sein de l'axe Étude des mobiliers. Nous avons lancé un protocole d'analyses chimiques (Y.Waksman) et pétrographiques (N.Cantin) sur les amphores Agora G199 découvertes dans plusieurs sites lyciens et pisidien (Limyra, Létôon et Sagalassos). L'analyse des amphores présentées dans cet article avait été envisagée dès le début du programme Xanthiaca, mais en raison de la suppression de la Mission Française de Xanthos, cette démarche n'a pas pu être menée à bien pour le moment.

87. Lund 2000, p.573, et Autret 2012, p. 254. 
sur les parois internes de certains exemplaires trouvés en mer $^{88}$. Pline l'Ancien mentionne en effet après le passum de Crète, ceux de Cilicie et d'Afrique ${ }^{89}$. Le vin chypriote apparaît également chez les auteurs anciens ${ }^{90}$. Enfin, une inscription peinte découverte sur une amphore de Pompéi mentionne le Passum Lycium ${ }^{91}$. Des indices convergents semblent indiquer que les trois régions : Chypre, la Cilicie et la Lycie (si l'origine lycienne de certaines amphores Agora G199 se trouvait confirmée) possédaient donc une forme commune de conteneur, témoignant par là même d'une certaine forme de communauté de culture et d'économie entre ces trois régions à l'époque romaine autour d'un produit régional spécifique: le passum.

Le vin constitue le produit transporté dans plus de $80 \%$ des amphores identifiées au sein de l'assemblage, celui de Cnide étant de loin le mieux représenté. Malgré des données quantitatives faibles, quelques individus, rappelons-le, les amphores Agora G199 découvertes dans le sanctuaire de Létô illustrent, par la diversité de leur origine, le dynamisme économique de la zone sud anatolienne à l'époque impériale et notamment la diffusion des vins chypriotes ${ }^{92}$ et ciliciens dans un schéma, qui, s'il est bien ancré régionalement, dépasse largement ce cadre pour atteindre le Levant et l'Égypte et également des marchés plus occidentaux.

\section{Conclusion}

D'un point de vue chronologique, l'assemblage présenté ici est comparable à ceux datés des $\mathrm{I}^{\mathrm{er}}$ et $\mathrm{II}^{\mathrm{e}}$ siècles à Anemurium en Cilicie (groupes A1 à A4), qui comprennent aussi des éléments résiduels du $\mathrm{I}^{\text {er }}$ siècle av. J.-C. ${ }^{93}$. Cependant, les assemblages de ce site contiennent une plus grande quantité de sigillées chypriotes que de sigillées orientales A, et la part des sigillées chypriotes augmente encore dans les ensembles du III $^{\mathrm{e}}$ siècle apr. J.-C. ${ }^{94}$. Cette constatation donne déjà un aperçu des échanges entre Chypre et la côte sud anatolienne à l'époque romaine. D'après l'assemblage du Létôon, où la part des sigillées chypriotes reste nettement

88. Autret 2012,p. 256.

89. Pline l'Ancien, Hist. Nat., XIV, 81 .

90. Voir sur le sujet Pline l'Ancien, Hist. Nat., XIV, 74, et plus largement Lund 2000, p. 572 .

91. CIL, IV, 5594, cité par A. Tchernia dans TCHERNIA 1986, Appendice VI.

92. Voir les travaux menés par J.Lund sur le sujet Lund 1999, fig. 10, et Lund 2000, p.572-573.

93. WiLliams 1989, p.2 et 105 à 107.

94. Williams 1989, p. 105. 
inférieure à celles des sigillées orientales A (respectivement $6 \%$ et 15\%), ces dernières constituant aussi un modèle pour la production locale de vaisselle fine, l'intégration dans la production locale, aux côtés des autres influences, montre l'importance des échanges entre Chypre et la Lycie. Il semblerait toutefois que ces relations se manifestent différemment d'ouest en est sur la côte sud-anatolienne.

En outre, les importations de vaisselle chypriote (6\% avec le groupe à pâte calcaire), qui restent inférieures aux importations rhodiennes (11\%) et cnidiennes (14\%) constituaient sans doute les compléments de charge des cargaisons d'amphores. En effet, près de $80 \%$ des récipients amphoriques identifiés du lot sont des conteneurs à vin, dont la majorité est originaire de la côte égéenne, comprise entre Rhodes au sud et Chios au nord. Parmi les vins importés, celui de Cnide est de loin le mieux représenté et semble indiquer un lien économique fort entre la Lycie et cette cité.

La composition des assemblages semble montrer que les échanges entre Chypre et la Lycie devaient s'effectuer de manière triangulaire, en incluant la péninsule cnidienne et Rhodes (Fig. 15). La proximité géographique d'une part, la tutelle commune lagide au début de l'époque hellénistique, ainsi que leur alliance précoce avec Rome d'autre part, ont certainement favorisé l'établissement de relations économiques entre Rhodes et la Lycie, et depuis cette zone vers le restant de la Méditerranée ${ }^{95}$.

Si l'étude de la circulation des produits céramiques, à l'échelle régionale, confirme le rôle prépondérant du trafic de cabotage dans les échanges économiques ${ }^{96}$, ces résultats illustrent aussi une organisation des échanges fondée sur un commerce de biens de consommation courante (grain, vin, bois par exemple), qui fonctionne grâce à l'établissement de vastes entrepôts ${ }^{97}$ dans des lieux possédant «un port construit dans une bonne situation commerciale ${ }^{98}$. C'est pourquoi, dès l'époque hellénistique, le rôle de port comme Caunos ${ }^{99}$ ou Patara, ou d'un site de mouillage comme celui de Castellorizo (Megisté) ${ }^{100}$,

95. Pour l'époque hellénistique, on connaît une mention du trafic maritime reliant la Lycie à Chypre, puis à l'Égypte. Cf. RoBERT 1987, p.508: «(...) les caïques, chargés des bois de Caunos, transportent leur cargaison sur les grands navires qui porteront le bois en Égypte. Ceux-ci étaient armés par des marins de Castellorizo, l'île si active alors de la côte sud de la Lycie, qui assuraient le trafic entre ces côtes anatoliennes, Chypre, la Syrie et l'Égypte».

96. Rougé 1966, p.84.

97. Rougé 1966, p. 419 .

98. Rougé 1966, p.38.

99. Cf. note 3 .

100. ROBERT 1962, p.56, note 3. Castellorizo / Mégisté appartient à la Pérée rhodienne et en constitue la limite orientale pendant la période hellénistique, faisant sans doute 


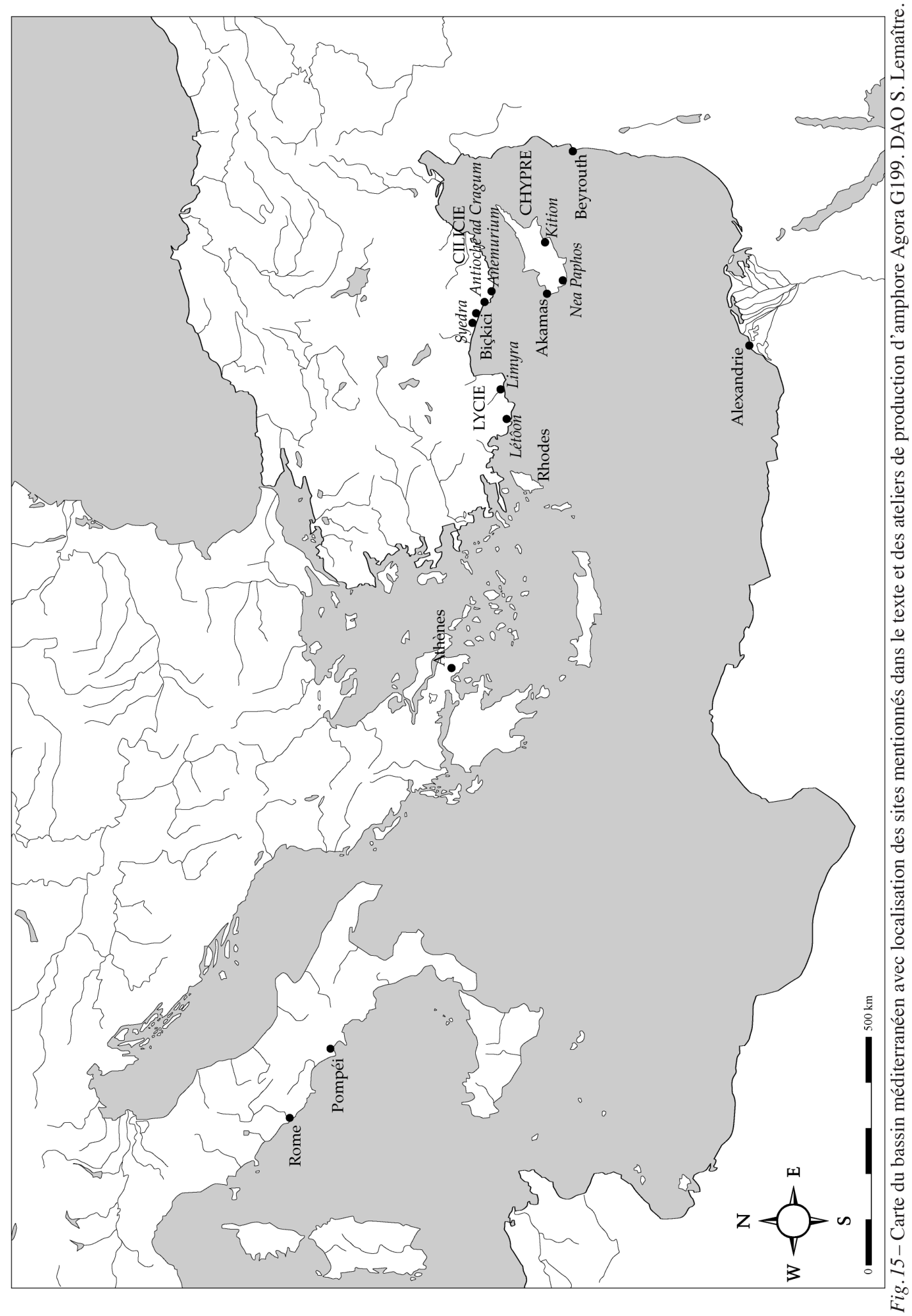


pourrait avoir été déterminant pour l'approvisionnement des sites lyciens, grâce à des réseaux d'échanges régionaux triangulaires, dans le cadre d'une navigation segmentée ${ }^{101}$.

\section{S. ÉLAIGNE}

HiSoMA, UMR 5189, Maison de l'Orient et de la Méditerranée

S. LEMAÎTRE

HeRMA, EA 3611, Université de Poitiers.

\section{Bibliographie}

AlPÖZEn T.O., B. BerkaYA, A. H. ÖDAş 1995, Commercial amphoras of the Bodrum Museum of underwater Archaeology, Ankara.

ARnaud P. 2005, Les routes de la navigation antique. Itinéraires en Méditerranée, Paris.

Arnaud P. 2011, «La Lycie et la Carie du Stadiasme», Anatolia Antiqua 19, p.411-432.

Autret C. 2012, «Cyprus and Cilicia: amphora production, trade and relations in the early Roman era», in A. Georgiou (éd.), Cyprus. An Island Culture, Society and Social Relations from the Bronze Age to the Venetian Period, Oxford, p.251-267.

Autret C. et N. Rauh 2010, «Roman Amphora production in Western Rough Cilicia», in Ü.AydinoĞLu, A.K.ŞEnol (éds), Olive Oil and Wine Production in Anatolia during Antiquity, Mersin Symposium Proceedings, Istanbul, p. 109-122.

BURKALTER F. 1987, «La céramique hellénistique et romaine du sanctuaire d'Aphrodite à Amathonte », $B C H$ 111, p.353-395.

Charlesworth M.P. 1961, Trade-Routes and Commerce of the Roman Empire, Hildesheim.

Davesne A. 2000, «La région des portiques du Létôon de Xanthos», CRAI, p.615-631.

Des Courtils J. 2007, «Introduction. La Lycie dans l'Histoire», in S.Lemaître (dir.), Céramiques antiques en Lycie (VII s. a.C. - VII s. p.C.). Les produits et les marchés, Ausonius Études 16, Bordeaux, p. 11-23.

Élaigne S. 2000, «Imitations locales de céramiques fines importées: le cas des colourcoated ware dans les contextes hellénistiques d'Alexandrie», Cahiers de la Céramique Égyptienne 6, p.99-112.

ÉLAIGNE S. 2002, «L'introduction des céramiques fines du bassin oriental de la Méditerranée à Alexandrie: importations et imitations locales », in J.-Fr. SALLES (éd.), Céramiques hellénistiques et romaines: productions et diffusions en Méditerranée orientale (Chypre, Égypte et côte syro-palestinienne), TMO 35, Lyon, p. 159-173.

ÉLAIGNE S. 2007, «Les importations de céramique fine hellénistique à Beyrouth (site BEY 002) : aperçu du faciès nord levantin», Syria 84, p. 107-142.

office de relais pour les échanges rhodiens orientés vers le sud-est anatolien et le Levant, puis l'Égypte, si l'on suit le sens des courants et des vents dont les navires de commerce étaient tributaires. Voir, pour Castellorizo, ARnaud 2005, p.216, et ARnaud 2011, p.424-425.

101. Arnaud 2005, p. 107-126. 
Élaigne S. 2012, La vaisselle fine de l'habitat alexandrin, Études Alexandrines 21, Le Caire.

Hayes J.W.1983, «The Villa Dionysos Excavation, Knossos: the pottery», Annual of the British School at Athens 78, p.97-170.

HaYes J.W. 1985, «Sigillate orientali», Atlante delle forme ceramiche II : ceramica fine romana nel bacino mediterraneo, Enciclopedia del'Arte Antica, p.1-95.

Hayes J.W. 1991, Hellenistic and Roman Pottery, Paphos III, Nicosie.

Holleaux M. 1893, «Notes sur l'épigraphie et l'histoire de Rhodes », BCH 17, p.52-69.

Lemaître S., S. Élaigne et A.Alary 2004, «Le mobilier céramique d'un remblai romain dans le sanctuaire du Létôon de Xanthos », Anatolia Antiqua XII, p.329-333.

LEMAîTRE S. 2006, «La Lycie et les échanges commerciaux dans l'Antiquité: les données du mobilier amphorique. Étude préliminaire», in Proceedings of the IIIrd International Symposium on Lycia 07-10 november 2005, Antalya, p. 385-399.

LEMAître S. 2007, «Vaisselle antique à Limyra en Lycie orientale (SO 3A et 3B)», in Th.Marksteiner, S.Lemaître, B. Yener-Marksteiner, «Die Grabungen am Südtor von Limyra», Österreichische Jahrhefte des archäologisches Institutes in Wien 76, p. 197-235.

Lund J. 1997, «The Distribution of Cypriot Sigillata as Evidence of Sea-trade involving Cyprus», in S.Swiny, R.Hohlfelder et H.Swiny (éds), Res Maritimae: Cyprus and the Eastern Mediterranean from Prehistory to Late Antiquity, Proceedings of the second international symposium "Cities on the Sea" (Nicosia, October 1994), Atlanta, p. 201-215.

Lund J. 1999, «Trade pattern in the Levant from ca. 100 BC to AD 200 as reflected by the distribution of ceramic fine wares in Cyprus», Münstersche Beiträge zur Antiken Handelsgeschichte 18, p.1-22.

Lund J. 2000, «The "pinched-handle" transport amphorae as evidence of the wine trade of Roman Cyprus », in Acts of the Third International Congress of Cypriot Studies, Nicosia, 16-20 avril 1996, Nicosie, p.565-578.

Marangou-Lerat A. 1995, Le vin et les amphores de Crète, Études Crétoises 30, Athènes.

MARQuí́ S. 2002, «La circulation des sigillées d'époque impériale au sud de Chypre», in J.-Fr. SAlles (éd.), Céramiques hellénistiques et romaines : productions et diffusions en Méditerranée orientale (Chypre, Égypte et côte syro-palestinienne), TMO 35, Lyon, p.289-301.

MARQUiÉ S. 2004, Production et circulation des céramiques fines d'époques hellénistique et romaine en Méditerranée: le cas des sigillées découvertes à Kition (Chypre), thèse de doctorat inédite sous la direction de M. Yon, Université Lyon 2.

Metzger H. 1978, «Fouilles du Létôon de Xanthos (1962-1972)», Proceedings of the $X^{\text {th }}$ International Congress of Classical Archaeology, Ankara, p. 789-803.

Meyza H. 1995, «Cypriot Sigillata and Cypriot Red Slip Ware: problems of origin and continuity», Hellenistic and Roman Pottery in the Eastern Mediterranean, IInd Nieborow Pottery Workshop, Varsovie, p. 179-202.

Meyza H. 2002, «Cypriot sigillata and its hypothetical predecessors», in J.-Fr. SALLES (éd.), Céramiques hellénistiques et romaines: productions et diffusions en Méditerranée orientale (Chypre, Égypte et côte syro-palestinienne), TMO 35, Lyon, p. 23-31. 
PANella C. 1973, «Appunti su un gruppo di anfore della prima, media e tarda età imperiale », Ostia III, Studi Miscellanei 21, p.460-633.

Panella C. 1986, «Oriente ed Occidente: considerazioni su alcune anfore "egee" di età imperiale a Ostia», $B C H$ Suppl. 13, p. 609-636.

Papuci-Wladyka E. 1995, «Hellenistic Pottery from Nea Paphos - First results of scientific analysis», Hellenistic and Roman Pottery in the Eastern Mediterranean, IInd Nieborow Pottery Workshop, Varsovie, p. 247-275.

PETIT Th. 1995, «Remarques sur la céramique "subclassique" et hellénistique, à vernis noir et à engobe du "Palais" d'Amathonte ", Hellenistic and Roman Pottery in the Eastern Mediterranean, IInd Nieborow Pottery Workshop, Varsovie, p.276-298.

PiCON M. et Fr. BLONDÉ 2002, «Les résultats d'un programme d'analyses effectué sur l'île de Chypre», in J.-Fr. SALles (éd.), Céramiques hellénistiques et romaines : productions et diffusions en Méditerranée orientale (Chypre, Égypte et côte syro-palestinienne), TMO 35, Lyon, p. 13-21.

RAUH N.K. 2004, «Pirated Knock-offs: Cilician Imitations of Internationally Traded Amphoras », in J.EIRING et J.Lund (éds), Transport Amphorae and Trade in the Eastern Mediterranean, Acts of the International Colloquium at the Danish Institute at Athens, September 26-29, 2002, Athènes, p. 329-336.

Rauh N. et C.W. Slane 2000, «Possible amphora kiln sites in Western Rough Cilicia», JRA 13, p.319-330.

RILEY J.A. 1979, «The coarse pottery from Benghazi», Excavations at Sidi Khrebish Benghazi (Berenice) II, Libya Antiqua Suppl. 5, p. 112-236.

Robert L. 1962, Villes d'Asie Mineure : études de géographie ancienne, Paris.

Robert L. 1987, Documents d'Asie Mineure. BEFAR 239 bis, Paris.

Robinson H.S. 1959, Pottery of the Roman Period. Chronology, The Athenian Agora V, Princeton.

Rougé J. 1966, Recherches sur l'organisation du commerce maritime en Méditerranée sous l'Empire Romain, Paris.

Russell J. 1986, «Excavations at Anemurium (Eski Anamur), 1985», Échos du Monde Classique 30, p. 173-183.

SALLes J.-Fr. 1993 (dir.), Les niveaux hellénistiques, Kition-Bamboula IV, Paris.

SCHNEIDER G. 2000, «Chemical and mineralogical studies of late Hellenistic to Byzantine pottery production in the eastern Mediterranean », Rei Cretariae Romanae Fautorum Acta 36, p.525-536.

TCHERNIA A. 1986, Le vin de l'Italie romaine. BEFAR 261, Rome.

Williams C.H. 1989, Anemurium. The Roman and Early Byzantine Pottery, Subsidia Mediaevalia 16, Pontifical Institute of Medieval Studies, Toronto.

Yener-Marksteiner B. 2007, «Keramik aus der Sondage 5 in der Westadt von Limyra», in Th.Marksteiner, S.Lemaître, B.Yener-Marksteiner, «Die Grabungen am Südtor von Limyra», Österreichische Jahrhefte des archäologisches Institutes in Wien 76, p. 197-235.

Zemer A. 1978, Storage jars in ancient sea trade, Haïfa. 